\title{
Public Transportation Management Services by Integrating GSM-GPS
}

\author{
Raghava Bhonagiri ${ }^{1}$, Prof.K.Subba Rao ${ }^{2}$, Vinod Kumar Varkala ${ }^{3}$, \\ Gopikrishna Siliveri ${ }^{4}$ \\ ${ }^{1,2}$ (Electronics\& Communication Engineering, DVR College of Engineering and Technology, Hyderabad, India) \\ ${ }^{3}$ (Electronics\& Communication Engineering, TRR College of Engineering and Technology, Hyderabad, India) \\ ${ }^{4}(R \& D$ Embedded Systems, CMC Limited, Hyderabad, India)
}

\begin{abstract}
This paper proposes and implements a solution for enhancing public transportation management services based on GPS and GSM in Andhra Pradesh. The system consists of four modules: BUS Station Module, In-BUS Module, BASE Station Module and BUS Stop Module. Equipped with PC and GSM modem, BUS Station Module sends the initialization information containing the bus number and license plate number to In-BUS Module and BASE Station Module using SMS. The microcontroller based In-BUS Module consisting mainly of a GPS receiver and GSM modem then starts transmitting its location and number of passengers to BASE Station Module. BASE Station Module equipped with a microcontroller unit and GSM modems interfaced to PCs is designed to keep track record of every bus, processes user request about a particular bus location out of BUS Station and updates buses location on bus stops. BUS Stop Module is installed at every bus stop and consists of a GSM modem, memory unit and dot matrix display all interfaced to a microcontroller. This module receives buses location information coming towards that stop from BASE Station module and displays the information on a dot matrix display. The results have shown that the developed system is useful for facilitating people using public transportation services.
\end{abstract}

Keywords : GPS;GSM;public transportation management services; Bus Station Module; In-BUS Module; BASE Station Module;BUS Stop Module;rush statistical analysis.

\section{INTRODUCTION}

In this paper, a transportation management system is developed for enhancing public transportation services based on integration of GPS and GSM. GPS is used as a positioning device while GSM is used as communication link between different modules. These modules include BUS Station Module, In-Bus Module, BASE Station Module and BUS Stop Module. Bus Station Module contains a GSM engine interfaced to PC and transmits the bus index and its license plate number to BASE Station. At the same time, it turns on GPS receiver installed in the bus. The bus then starts transmitting its location to the BASE Station. The BASE Station comprises of a GSM engine interfaced to a microcontroller for processing user request of bus location as well as a number of other GSM engines interfaced to various PCs each reserved for a separate bus to update the location information of that bus. The buses location data from BASE Station is sent to each bus stop. BUS Stop Module after receiving buses location data through GSM engine displays it on dot matrix display installed at each bus stop. The block diagram of the proposed system is shown in Fig. 1.

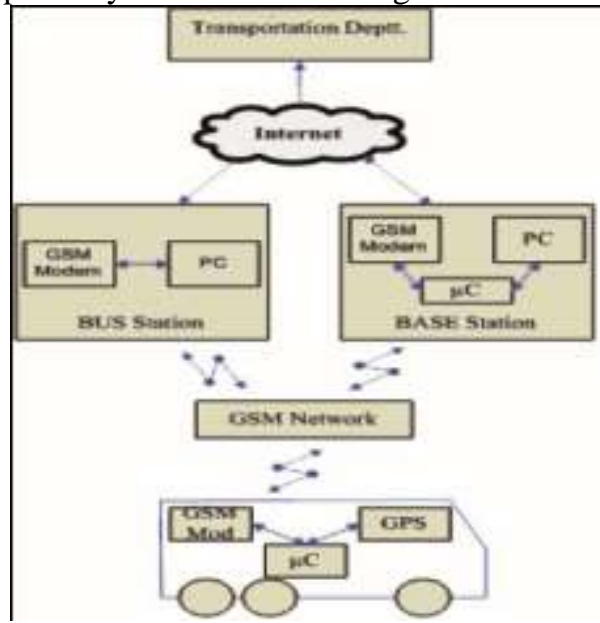

Figure 1. Block Diagram of Transportation Management System 


\section{HARDWARE SPECIFICATIONS}

The following hardware components are used in building the entire system:

\section{A. GPS Receiver}

In order to keep track record of bus, a Garmin GPS35 receiver, powered from the bus main battery, is installed in each bus. The Garmin GPS35 is a complete GPS receiver and embedded antenna designed for a broad spectrum of OEM system applications. The GPS35 tracks up to twelve satellites at a time while providing one-second navigation updates and low power consumption. Its far-reaching capability meets the sensitivity requirements of land navigation as well as the dynamics requirements of high-performance aircraft. Internal memory backup allows the GPS35 to retain critical data such as satellite orbital parameters, last position, date, and time.

\section{B. GSM Modem}

A wireless link between the modules is provided with Nokia 12i GSM module. Nokia 12i offers advance GSM connectivity and supports EDGE/GPRS and HSCSD with automated GSM connection establishment It is equipped to provide reliable remote connections and offers application level watchdogs, inbuilt self check mechanisms and a reliable Virtual Machine (VM) for JAVA. Nokia 12i also supports reliable inbuilt internet protocols: TCP/IP for reliable data transfer, UDP/IP for audio and video streaming and HTTP for accessing web pages. The module can also be connected to an external GPS device that supports National Marine Electronics Association (NMEA) standard. The inbuilt NMEA parser can parse the location data from the output that it receives from the GPS device. External microcontroller can use AT commands to communicate with Nokia $12 \mathrm{i}$ and simple remote I/O applications can easily be controlled via text messages.

\section{Microcontroller}

AT89C52 microcontroller is selected because it is a powerful microcomputer which has low power consumption and provides a highly flexible and cost-effective solution to many embedded control applications. It has $8 \mathrm{~K}$ bytes of in system reprogrammable flash memory, 256 bytes of internal RAM, 32 programmable I/O lines, three 16 bit timers/counters, eight interrupt sources and a programmable serial channel.

\section{Memory}

256K Nonvolatile RAM (NV-Ram) DS1230Y-85 is used for storing data in In-BUS Module (in case of sparse GSM coverage) and at BUS Stop Module for displaying on dot matrix display. NV-RAM is selected because it combines the best of RAM and ROM: the read and write ability of RAM and non-volatility of ROM. The DS1230 Nonvolatile SRAM is 262,144-bit, fully static, nonvolatile SRAM organized as 32,768 words by 8 bits. Each NV SRAM has a self-contained lithium energy source and control circuitry which constantly monitors VCC for an out-of-tolerance condition. When such a condition occurs, the lithium energy sources is automatically switched on and write protection is unconditionally enabled to prevent data corruption.

\section{E. Battery Backup}

In-Bus Module is provided with an internal battery so that whenever power from main battery is disconnected, microcontroller continues to transmit the location to BASE station. A message is also sent to BASE station to notify it about the disconnection of main battery. When the power is resumed, the internal battery begins to recharge.

\section{F. Alarms}

The microcontroller unit in In-BUS Module sends different alarm signals for different events to BASE Station Module.

1) On Backup Battery: When the main battery is switched off, a notification is sent to BASE station.

2) Stoppage: When the bus is stationary for more than a specified time, BASE station is informed by a stoppage alarm. In case of an accident or any other fault occurred in bus, the driver can notify the BASE station by pressing a button in bus.

3) Getting Late: When the bus is not covering a certain distance in a defined range of time, an alarm signal of getting late is sent to BASE station.

4) Route Deviation: When the bus deviates from the assigned route by a given margin, BASE station is notified. 


\section{SYSTEM MODULES AND NETWORK OPERATION}

The entire system/network comprises of four modules: BUS Station Module, In-BUS Module, BASE Station Module and BUS Stop Module. The working and interconnection of these modules is described in this section.

\section{A. BUS Station Module}

BUS Station Module is installed at bus terminals from where the bus will depart. It contains a LASER and a GSM modem connected to a PC. When the bus enters the terminal pad, it is detected by the LASER sensor. The operator at the terminal enters the license plate number in database. A count number is then accordingly assigned to the bus e.g., bus leaving the terminal first will be assigned a number 1 . The route number of bus along with the direction information, assigned count number and license plate number is sent to the BASE Station via GSM. An example of the transmitted header is of the form "33U01LZR7240" where ' 33 ' is the bus route number. Transportation Department, ' $U$ ' is upward direction of bus (' $D$ ' will be downward direction), ' 01 ' is the count number assigned to the bus and 'LZR7240' is license plate number of bus. An 'ON' signal is also transmitted to the In-BUS Module installed in the bus for initialization. The flow chart of module software is shown in Fig. 2.

\section{B. In-BUS Module}

In-BUS Module is installed inside every bus and consists of a GPS receiver, a GSM modem, a NVRAM, infrared object counting sensors, door opening/closing sensors and an emergency button; all interfaced to AT89C52 microcontroller. After receiving the initialization signal form BUS Station Module, this module starts transmitting bus location to the BASE Station. At each stop, when the driver opens the door, an interrupt is generated and microcontroller starts counting the numbers of passengers entering and leaving the bus with the help of infrared sensors. This count value on per stop basis is transmitted to the BASE Station. In case of an emergency situation (e.g., when fault occurs in bus), driver can press the emergency button to inform BUS and BASE Station units about the location of bus. The BUS station operator can then adjust the schedule accordingly and send an additional bus for facilitating the passengers. Microcontroller present in this module continuously calculates the difference in consecutive GPS locations. If the difference remains near zero for more than a designated time, then a getting late message is transmitted to the BUS and BASE stations. In case of sparse GSM coverage, location information is stored in non-volatile RAM. After regaining the GSM network, previous locations are updated to the BASE station. The block diagram for this module is shown in Fig. 3 while flow chart of module software is shown in Fig. 4.

\section{BASE Station Module}

This module is the central part of the network. It accepts location information of buses through respective GSM modems and maps the information on Google Map for visualization. It also receives the number of passengers entering and leaving the bus on per stop basis from In-BUS Module for statistical analysis. The message received is of the form " $20,10,2345.3522 \mathrm{~N}, 09022.0288 \mathrm{E}$ ". The first two strings denotes the number of passengers entering and leaving the bus respectively and next two strings denotes the location information; all separated by commas. Another GSM modem is used to get the user request of location information of a particular bus. An example of the query put by the passenger is of the form " $33 \mathrm{U}$ " or " $33 \mathrm{U} 10$ ". In first instance i.e., " $33 \mathrm{U}$ ", ' 33 ' is the route number and ' $U$ ' designates the direction flag while in second instance i.e., "33U10", additional digit '10' denotes the bus stop number where the passenger is standing. The microcontroller attached with this GSM modem passes on the user request to the PC dedicated for that route number. The PC after processing the request data sends desired location information in form of bus stop name to microcontroller. The microcontroller then transmits this information back to the user. The information that passenger will receive contains the location of all buses out of terminal in desired direction in former query while in case of later query, he will get the location of those buses which are coming towards the particular bus stop number in desired direction along with time information. The time information is embedded in message to account for any delay in processing the user request. An example of the information received by the user is of the form "N.G College, Clock Tower, V.T Colony, Srinagar colony- 12:30 P.M." where first four strings are bus stops names telling where the buses are currently followed by the time on which the location information is get from the map and message is sent to user. BASE station also monitors the emergency situations transmitted from In-BUS Module. In addition to this, the station keeps record of security issues and traffic congestion conditions and directs the driver to change the route if desired. The block diagram of the module is shown in Fig. 5 while module software is shown in Fig. 6. 


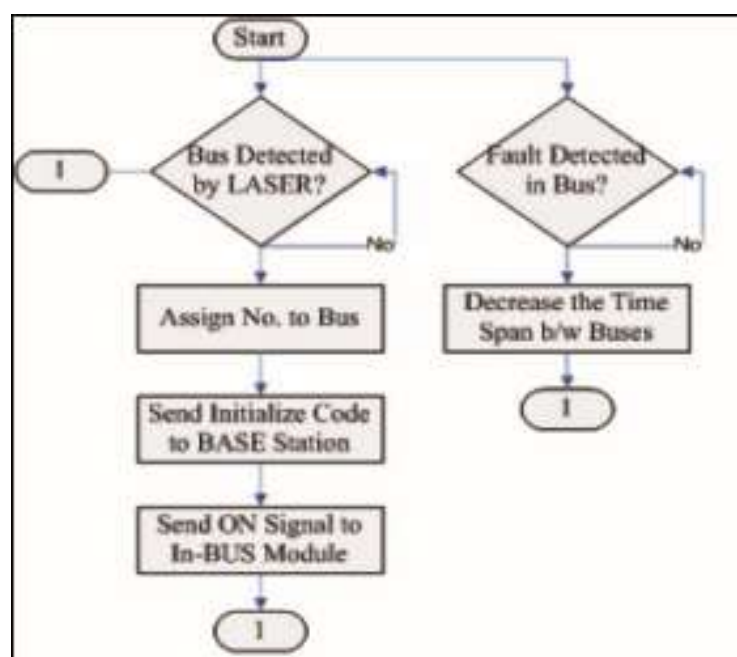

Figure 2. Flow Chart of BUS Station Module

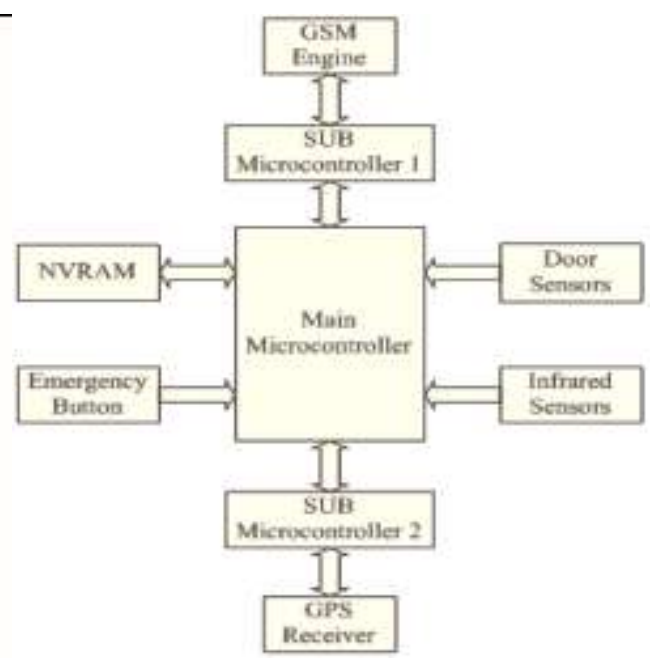

Figure 3. Block Diagram of In-BUS Module

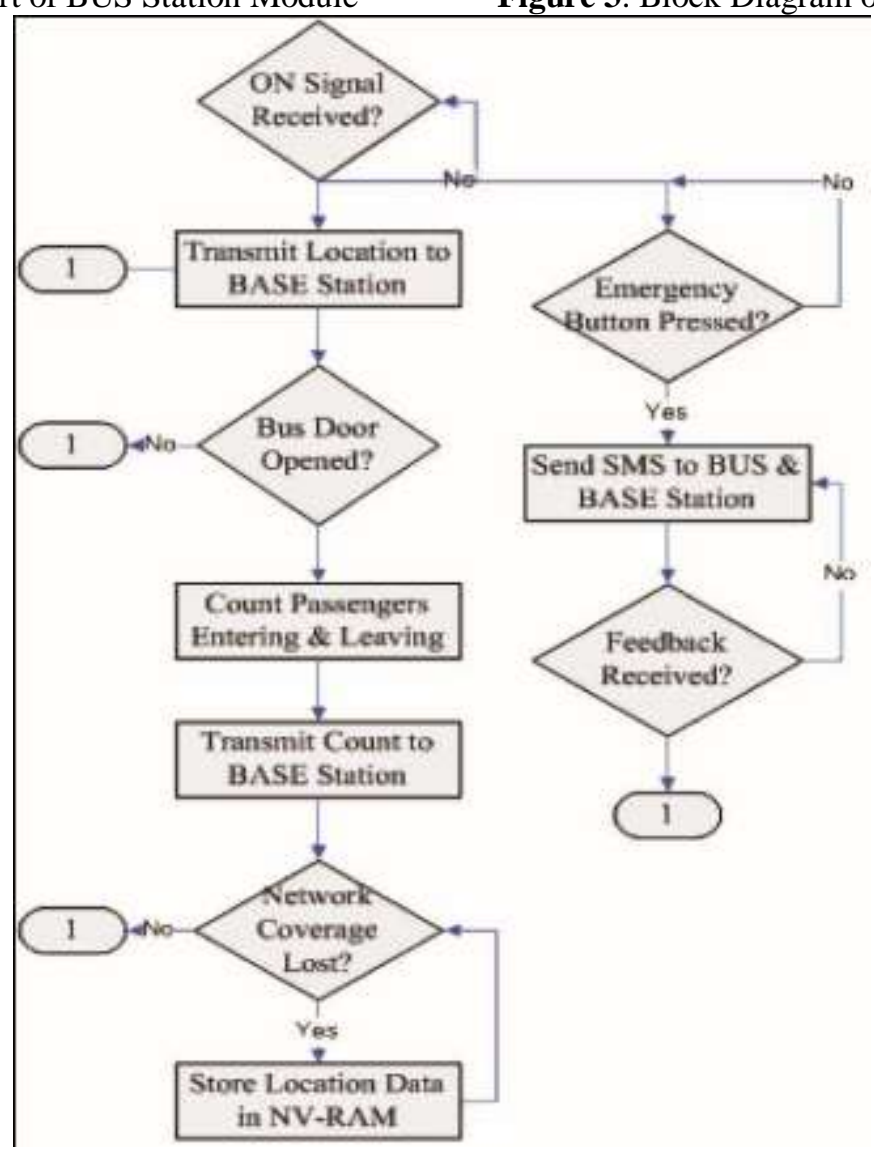

\section{BUS Stop Module}

Figure 4. Flow Chart for In-BUS Module

This module is installed at every bus stop to let the passenger know about the location of buses coming towards that stop. It comprises of a GSM modem, a NV-RAM and dot matrix display; all interfaced to 89C52 microcontroller. After receiving the bus location data in the form of stop names from BASE station, microcontroller stores it in non-volatile RAM. A sample message received by BUS Stop Module installed at 'N.G College Stop' is of the form "33, Clock Tower; 22, V.T Colony; 77, Srinagar colony. The message contains information of those buses only which will pass by the designated stop. First two digits of a sub-string denote the bus route number followed by the bus stop name which is the current location of bus coming towards the specified stop. Microcontroller after retrieving the stored information displays it on a 3x15 dot matrix display. The microcontroller refreshes the information with a rate of 10seconds. In case of an emergency situation, the location of next incoming bus is displayed. The block diagram of this module is shown in Fig. 7 and flow chart of module is shown in Fig 8. 


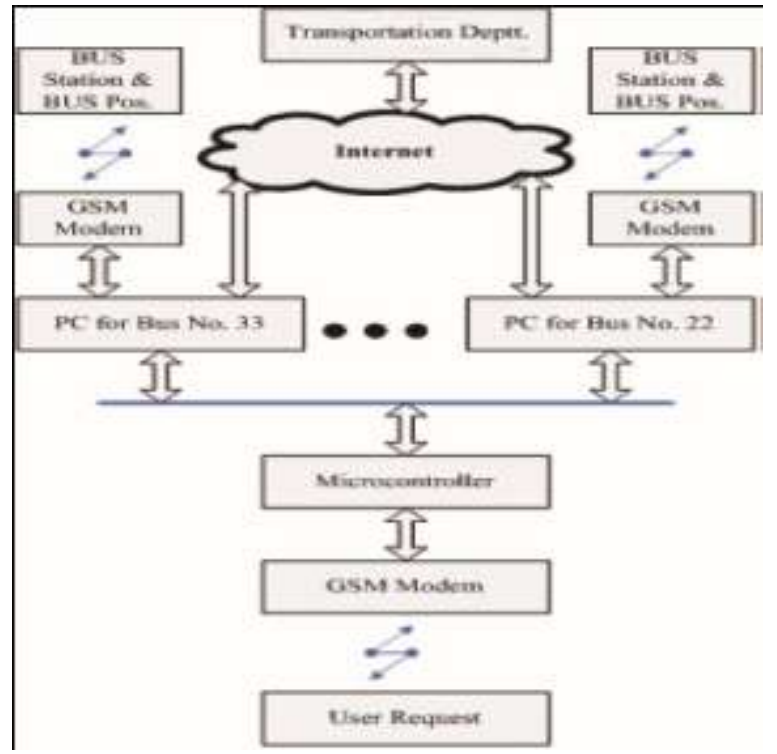

Figure 5. Block Diagram of BASE Station Module Module

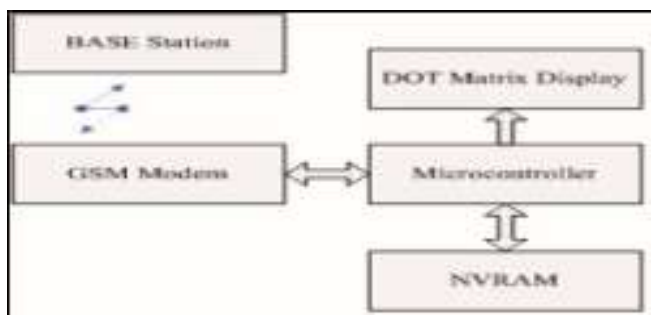

Figure 7. Block Diagram of BUS Stop Module

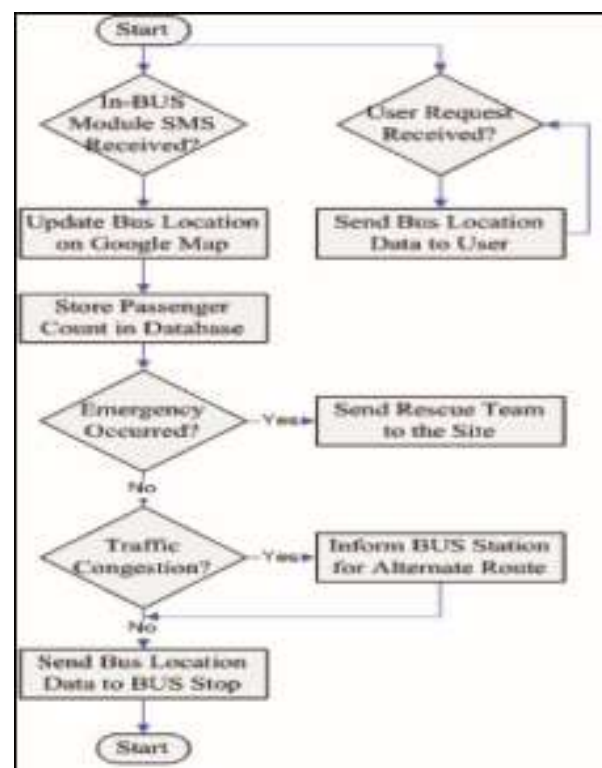

Figure 6. Flow Chart for BASE Station

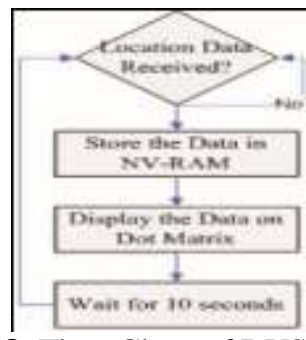

Figure 8. Flow Chart of BUS Stop Module

\section{SYSTEM SIMULATION RESULTS}

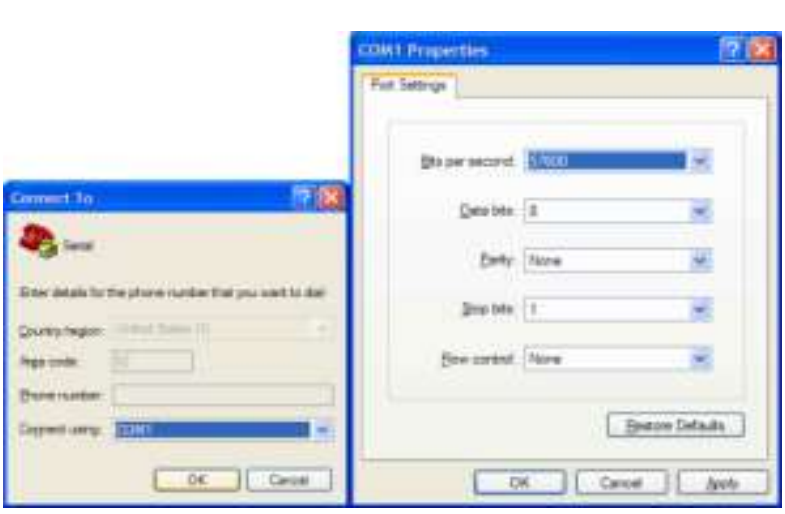

Serial Terminal Program Configuration Configuration

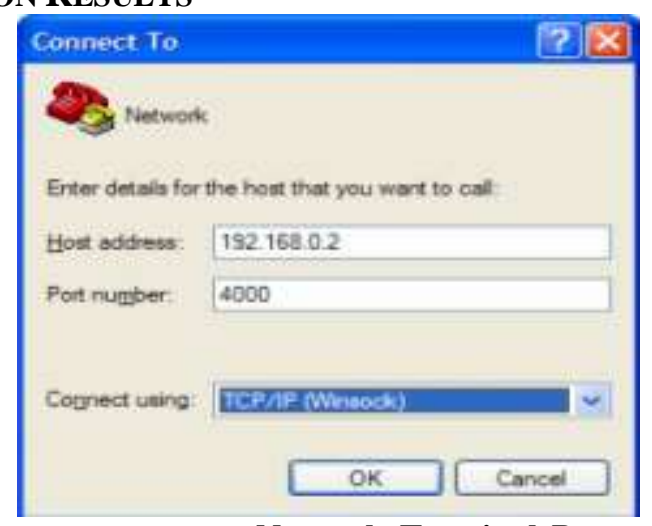

Network Terminal Program

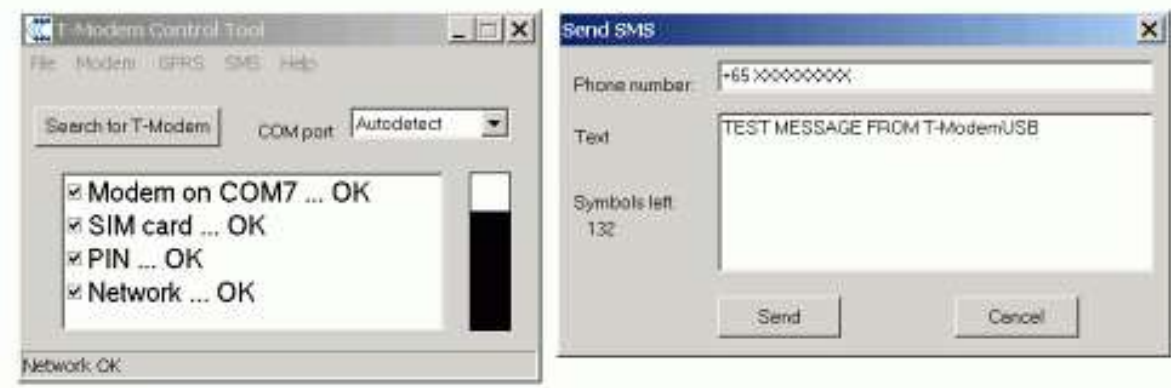

Windows based control panel to setup GSM modem, GPRS and send SMS 


\section{CONCLUSION}

In this paper, design and development of a low cost transportation management system based on integration of GPS and GSM data is described. The system comprises of various modules which are wirelessly linked with GSM modems. Cost effective SMS service of GSM network is used for the transfer of data between the modules. A new service, to facilitate the people who use public transport for traveling, is introduced inside the city. The service provides the user with current location information of desired buses based on which the user can adjust his schedule accordingly. The service therefore vanishes the need of waiting at the bus stop thus saving a lot of time. For the passengers not utilizing the service, displays are installed at bus stop to let them know the buses location coming towards that stop. The system is also efficient in handling the emergency situations e.g., in case some kind of technical fault occurred in bus, the operator at bus terminal is informed and the departure time between the buses is reduced.

\section{FUTURE WORK}

The system can be made automatic by installing cameras at bus terminals which can automatically read the license plate number of buses thereby eliminating the operator. An automatic route guider display can be installed in buses to better update the alternative route in case of serious road congestions. Fare collecting system can also be automated by providing another mobile service to which all the passengers using public transport are subscribed.

\section{REFERENCES}

[1] M.A.Mazdi,J.C.Mazdi,R.D.Mckinaly, The 8051 Microcontroller and Embedded Systems,Pearson education,2006

[2] Embedded Systems Design by Arnold S. Berger, 2001.

[3] The 8051 Microcontroller: Hardware, Software and Interfacing by James Stewart, Kai Mia.

[4] Internetworking with TCP/IP (VOL.I) Principles, Protocols and Architecture by Douglas Comer, 2000

[5] www.garmin.com/products/gps35

[6] www.mathworks.com

[7] www.d-d-s.nl/fotos-nokia/n12i.com

[8] www.alldatasheet.com

\section{BIOGRAPHIES}

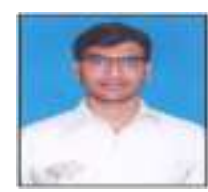

Raghava Bhonagiri, presently working as Assistant Professor in ECE department at DVR College of engineering and technology, Hyderabad. He has got B.tech degree from Swami Ramanantha Thirtha Institute of science and Technology Nalgonda, A.P. He has got M.tech (Embedded Systems) from same Swami Ramanantha Thirtha Institute of science and Technology Nalgonda, A.P.

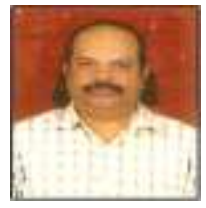

Prof.K. Subba Rao presently working as HOD in ECE department at DVR College of engineering and technology, Hyderabad. He has got B.tech degree from JNTU Hyderabad in1984. He has got M.tech degree from JNTU Hyderabad in1987. He has got PhD from IIT Kharagpur in1994. Previously he worked in CMC Hyderabad as Systems Engineer from 1984-1985. He worked as HOD in several colleges like Amaravathi University, Amaravathi, Auroras Engineering College, CVSR Engineering College, DRKIST in Hyderabad from 1988-2012.

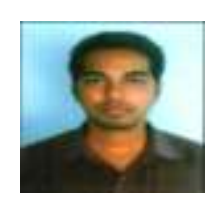

Vinod Kumar Varkala, presently working as Assistant Professor in ECE department at TRR College of Engineering and Technology, Hyderabad. He got B.Tech degree in Electronics and Instrumentation from Swami Ramanantha Thirtha Institute of science and Technology Nalgonda, A.P. He got got M.Tech degree (Embedded Systems) from Swami Ramanantha Thirtha Institute of science and Technology Nalgonda, A.P.He has 3 years of teaching experience.

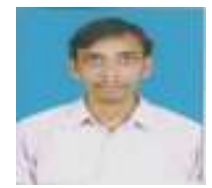

Gopikrishna Siliveri, presently working as IT-Engineer (Hardware/Embedded Systems) in R \&D department at CMC Ltd , Hyderabad .He is having hands on experience on complete Hardware Design life cycle and development in the field of Real time embedded systems, . He has got B.tech degree in Electronic and Instrumentation from Swami Ramanantha Thirtha Institute of science and Technology Nalgonda, A.P. 